34. Pellegrini JW, Lipton SA. Delayed administration of memantine prevents $N$-methyl-D-aspartate receptor-mediated neurotoxicity. Ann Neurol 1993;33:403-407.

35. Greenamyre JT, Eller RV, Zhang Z, Ovadia A, Kurlan R, Gash DM. Antiparkinsonian effects of remacemide hydrochloride, a glutamate antagonist, in rodent and primate models of Parkinson's disease. Ann Neurol 1994;35:655-661.

36. Uitti RJ, Rajput AH, Ahlskog JE, et al. Amantadine treatment is an independent predictor of improved survival in parkinsonism [abstract]. Can J Neurol Sci 1993;20(Suppl 4):S235.

\title{
Neurologic consequences of HTLV-II infection in injection-drug users
}

\author{
G. Dooneief, MD; R. Marlink, MD; K. Bell, MD; K. Marder, MD; B. Renjifo, MD, PhD; Y. Stern, PhD; and \\ R. Mayeux, MD
}

Article abstract-Several case reports have suggested an association between human T-cell lymphotropic virus type II (HTLV-II) infection and chronic neurologic disease. We performed serial neurologic examinations in injection-drug users (IDU), a group known to be at increased risk for HTLV-II infection. At baseline, those infected with HTLV-II alone, human immunodeficiency virus (HIV) alone, or both were significantly more likely to have neurologic disability than uninfected subjects. Longitudinally, HTLV-II infection was independently associated with the development of global neurologic disability and neuropathy, suggesting that HTLV-II causes neurologic disease.

NEUROLOGY 1996;46:1556-1560

Human T-cell lymphotropic virus type II (HTLV-II) was first isolated from the cells of a patient with an atypical hairy-cell leukemia, and it has also infected patients with other lymphoid malignancies. ${ }^{1}$ Similarly, HTLV-II has infected patients with chronic progressive neurologic disease. ${ }^{2}$ In the U.S., the principal reservoir of HTLV-II infection is the injection drug user (IDU) population. ${ }^{3}$ To determine the neurologic consequences of HTLV-II infection, we investigated neurologic findings in a cohort of IDUs tested for HTLV-I, HTLV-II, and HIV (human immunodeficiency virus), and followed for up to 4 years.

Subjects and methods. One hundred sixty-one IDUs in a longitudinal study of the neurologic and neuropsychologic effects of HIV infection were recruited as volunteers from a methadone maintenance clinic at St. Luke's-Roosevelt Hospital and from the infectious disease clinic at Harlem Hospital Center, both in New York City. All had used intravenous drugs at least 10 times in the 5 years preceding enrollment, were between the ages of 18 and 60 , and knew their HIV status prior to study entry. None of the subjects met 1986 criteria for AIDS, ${ }^{4}$ nor did any have a history of encephalopathy or chronic neurologic disorder unrelated to HIV infection (e.g., posttraumatic dementia, multiple sclerosis).

Subjects were seen biannually for up to 4 years. At each visit, subjects were examined by a neurologist shielded from their HTLV and HIV serostatus. The examination has been described in detail elsewhere. ${ }^{5}$ Individual items from the examination were grouped into nine larger categories representing functionally distinct parts of the neurologic examination (e.g., extrapyramidal signs, cranial nerve signs) and rated on a 5- or 6-point severity scale. For example, subjects with a score of 2 on the neuropathy item had a mild distal gradient of sensory impairment in at least three extremities and at least two sensory modalities (pain/temperature, light touch, vibration, position sense), but did not have a complete loss of sensation or any apparent disability as a result of the neuropathy. A summary measure of overall neurologic disability modified from the Kurtzke disability status scale for multiple sclerosis ${ }^{6}$ was derived from the severity and number of defective categories. The functional categories and the Kurtzke score were dichotomized at levels that neurologists would detect and consider clinically meaningful. The examination has good interrater reliability. ${ }^{7}$

Subjects were systematically interviewed about their drug and alcohol usage. Years of illicit drug use were dichotomized at the median value: "long," 15 years or more; "short," less than 15 years. Similarly, years of alcohol use were dichotomized: "long," 20 years or more; "short," less than 20 years. Drug and alcohol use variables were included in the analysis to control, albeit only grossly, for the

From the Gertrude H. Sergievsky Center (Drs. Dooneief, Bell, Marder, Stern, Mayeux), the departments of Neurology (Drs. Dooneief, Bell, Marder, Stern, Mayeux) and Psychiatry (Drs. Stern, Mayeux) at the College of Physicians and Surgeons and the HIV Center for Clinical and Behavioral Studies (Drs. Dooneief, Bell, Marder, Stern, Mayeux), Columbia University, New York, New York and from the Harvard AIDS Institute, Harvard School of Public Health (Drs. Marlink, Renjifo) Boston, MA.

Supported by center grant P50-MH43520 from the National Institute of Mental Health/National Institute on Drug Abuse to the HIV Center for Clinical and Behavioral Studies.

Presented in part at "Neuroscience of HIV infection: basic and clinical frontiers." Vancouver, British Columbia, Canada, 1994.

Received July 13, 1995. Accepted in final form November 10, 1995.

Address correspondence and reprint requests to Dr. George Dooneief, Sergievsky Center, 630 W. 168 th Street Box 16 , New York, NY, 10032. 


\begin{tabular}{|c|c|c|c|c|}
\hline & Uninfected & $\begin{array}{l}\text { HTLV-II } \\
\text { alone }\end{array}$ & HIV alone & Co-infected \\
\hline $\mathrm{N}$ & 56 & 13 & 61 & 26 \\
\hline Mean age* & 37.6 & 45.7 & 36.5 & 42.9 \\
\hline Mean educ & 11.9 & 11.5 & 11.8 & 11.7 \\
\hline$\%$ Female & $32 \%$ & $46 \%$ & $36 \%$ & $46 \%$ \\
\hline $\begin{array}{l}\% \text { African- } \\
\text { American* }\end{array}$ & $67 \%$ & $100 \%$ & $69 \%$ & $96 \%$ \\
\hline Mean CD4/mm3† & 1056 & 1095 & 407 & 505 \\
\hline \multirow[t]{3}{*}{ Neurologic disability } & $5 / 56$ & $5 / 13$ & $19 / 61$ & $11 / 26$ \\
\hline & $\mathrm{OR}=1$ & 6.7 & 4.1 & 6.9 \\
\hline & reference & $(1.5,29.3) \ddagger$ & $(1.4,11.8) \ddagger$ & $(2.1,23.0)$ 丮 \\
\hline \multirow[t]{2}{*}{$\begin{array}{l}\text { Cognitive } \\
\text { impairment }\end{array}$} & $\begin{array}{c}11 / 56 \\
\mathrm{OR}=1\end{array}$ & $\begin{array}{l}5 / 13 \\
2.1\end{array}$ & $\begin{array}{c}27 / 61 \\
2.3\end{array}$ & $\begin{array}{l}7 / 26 \\
1.2\end{array}$ \\
\hline & reference & $(0.6,7.9)$ & $(1.0,5.2) \ddagger$ & $(0.4,3.4)$ \\
\hline \multirow[t]{2}{*}{$\begin{array}{l}\text { Extrapyramidal } \\
\text { signs }\end{array}$} & $\begin{array}{c}2 / 56 \\
\mathrm{OR}=1\end{array}$ & $\begin{array}{l}2 / 13 \\
4.9\end{array}$ & $\begin{array}{l}1 / 61 \\
0.4\end{array}$ & $\begin{array}{l}5 / 26 \\
5.8\end{array}$ \\
\hline & reference & $(0.6,39.0)$ & $(0.04,4.7)$ & $(1.1,32.5)$ 丰 \\
\hline \multirow[t]{3}{*}{ Bladder dysfunction } & $0 / 56$ & $0 / 13$ & $2 / 61$ & $0 / 26$ \\
\hline & $\mathrm{OR}=1$ & 4.3 & 3.8 & 2.2 \\
\hline & reference & $(0.04,527)$ & $(0.1,114)$ & $(0.02,264)$ \\
\hline \multirow[t]{3}{*}{ Neuropathy } & $25 / 56$ & $9 / 13$ & $31 / 61$ & $18 / 26$ \\
\hline & $\mathrm{OR}=1$ & 3.1 & 1.2 & 2.3 \\
\hline & reference & $(0.8,12.9)$ & $(0.5,2.4)$ & $(0.9,6.4)$ \\
\hline
\end{tabular}

* HTLV-II seropositive and seronegative subjects significantly different at $p<0.05$ level.

$\dagger$ HIV seropositive and seronegative subjects significantly different at $p<0.05$ level.

$¥$ Significantly different from reference group at $p<0.05$ level.

possible effects of drug use and alcohol use on the occurrence of neurologic dysfunction.

Laboratory studies at each visit included complete blood count and lymphocyte typing. Initial blood samples for each subject were serologically analyzed for HTLV-I and HTLV-II by Western blots to whole cell lysates of either HUT 102 cells (HTLV-I infected), or C81/66 cells (HTLV-II infected). All positive or indeterminate sera were also tested by Western blotting to type-specific synthetic peptides to the envelope glycoproteins of HTLV-I (gp46); and HTLV-II (gp52). ${ }^{8}$ All sera able to be typed for HTLV-I or HTLV-II via these serologic tests were noted. For sera untypable by these means, peripheral blood mononuclear cells from the study subjects were used for polymerase chain reaction (PCR) analysis using type-specific primer pairs to HTLV-I or HTLV-II, as previously described. ${ }^{9}$ Sera reactive only to gag-related antigens by whole-cell lysate Western blotting and not definitively reactive to typespecific peptides or PCR analysis were not considered HTLV seropositive. Subjects were classified with respect to their HIV and HTLV-II serostatus as uninfected, infected with HTLV-II only, infected with HIV only, or co-infected.

One-way analysis of variance was used to compare continuous demographic variables (age, education, CD4 count) in the four groups. Odds ratios (OR) and $95 \%$ confidence intervals (CI) in the uninfected group were calculated by the method of Breslow and Day. Logistic regression was performed to determine the odds of neurologic disability, extrapyramidal signs, bladder dysfunction, stocking/glove neuropathy, or cognitive impairment in the infected groups relative to the uninfected group, while controlling for age, race, gender, drug and alcohol use, and, among the HIVseropositive subjects, CD4 count. The relative risks (RR) of developing neurologic disability, extrapyramidal signs, bladder dysfunction, stocking/glove neuropathy, or cognitive impairment among infected subjects over time were assessed by the Cox proportional hazards method, controlling for age, race, gender, drug and alcohol use, and CD4 count (among the HIV-seropositive subjects) and excluding prevalent cases (those with the abnormality at baseline) for each of the endpoints evaluated. Analyses were performed using SPSS/PC + (SPSS Inc., Chicago, IL, 1990).

Results. No subjects were seropositive for HTLV-I, but 39 of 156 (25\%) were seropositive for HTLV-II. Of these, 26 (67\%) were co-infected with HIV. Of the 117 HTLV-II seronegative subjects, $61(52 \%)$ were HIV seropositive. Demographic data for the four groups are presented in the table. Subjects infected with HTLV-II were significantly older 


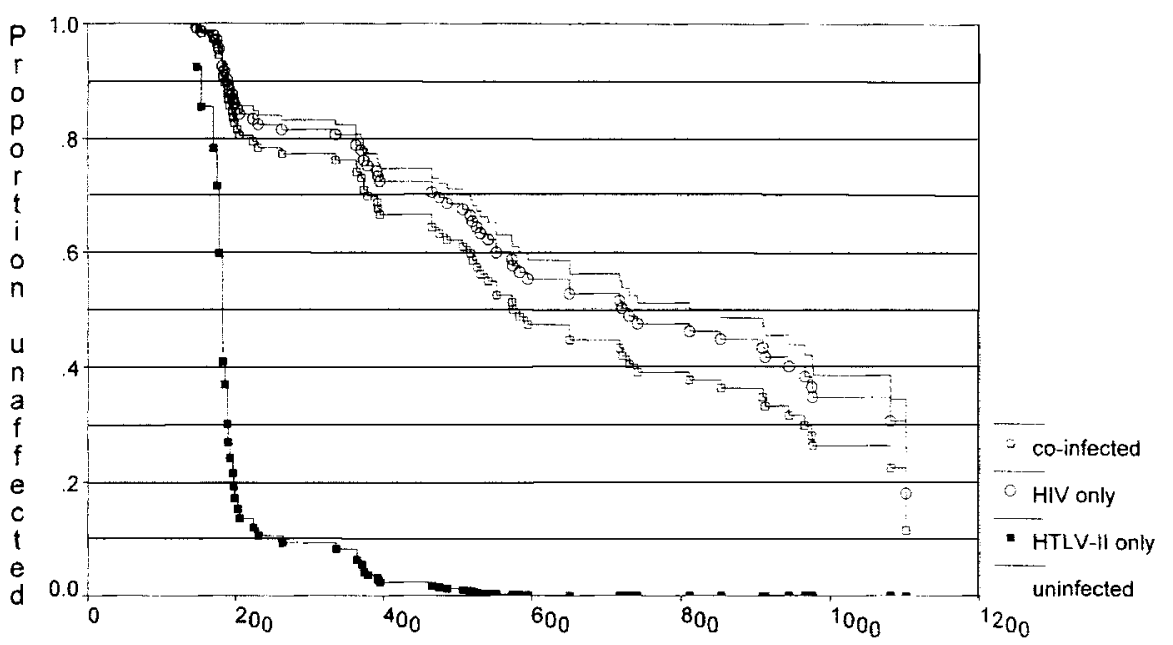

days from baseline to neurologic disability endpoint
Figure 1. Cox proportional hazards plot showing development of neurologic disability among members of a cohort of injection drug users during follow-up. (mean difference nearly 7 years, 95\% CI [4,9.5], $p=0.031$ ) and were more likely to be African-American $(\mathrm{OR}=16.3$, $95 \%$ CI $[3.4,78])$ than subjects who were not infected with HTLV-II. As expected, CD4 counts were significantly lower in the HIV-infected patients. Because the age difference may be a surrogate for other differences that are not easily controlled by including age in a multivariate analysis (e.g., a marker for duration of drug use or a cohort effect associated with secular changes in drug use), frequency-matched samples of subjects from the two HTLV-II seronegative groups (with and without HIV infection) with the same mean and range of ages as the respective HTLV-II seropositive groups were employed to verify the results of subsequent analyses from the full cohort.

A cross-sectional analysis of the independent and interaction effects of HTLV-II and HIV at the initial evaluation was performed (table). Of note, no subjects had spastic paraparesis. HTLV-II-seropositive, HIV-seropositive, and co-infected subjects were significantly more likely to have neurologic disability than uninfected subjects. HIV-seropositive subjects were significantly more likely to have cognitive impairment than uninfected subjects. While the frequency of cognitive impairment was $44 \%$ among HIVpositive subjects and $27 \%$ among co-infected subjects, this difference was not statistically significant. Co-infected subjects were significantly more likely to have EPS than uninfected subjects, but no interaction was observed. Repetition of these analyses with the subsample frequencymatched for age revealed similar results, except that the odds of extrapyramidal signs were no longer significantly increased among co-infected subjects.

The findings were also confirmed by logistic regression analysis of baseline clinical findings (controlling for age, race, gender, drug and alcohol use, and CD4 count in HIVseropositive subjects), which revealed increased overall neurologic disability among the HIV-seropositive (OR = $5.1,95 \%$ CI $[1.36,19.30], p=0.016)$ and co-infected subjects $(\mathrm{OR}=6.3,95 \%$ CI $[1.44,27.49], p=0.015)$, with a trend toward increased neurologic disability among the HTLV-II-seropositive subjects (OR $=4.4,95 \% \mathrm{CI}[0.88$, 21.39], $p=0.071$ ).

During follow-up 26 subjects died [two uninfected (4\%), one infected with HTLV-II alone (8\%), 17 infected with
HIV alone (28\%), and six co-infected (23\%)] and an additional 65 did not complete the full 4 years of follow-up [ 29 uninfected $(52 \%)$, six infected with HTLV-II alone $(46 \%)$, 25 infected with HIV alone $(41 \%)$, and five co-infected $(19 \%)]$. However, the mean duration of follow-up was not significantly different in the four subgroups (uninfected: 2.4 years, infected with HTLV-II alone: 2.4 years, infected with HIV alone: 2.3 years, and co-infected: 2.7 years).

In the longitudinal analysis of individuals without significant neurologic disability at baseline, Cox proportional hazards models demonstrated that the development of neurologic disability during follow-up was significantly increased in those with HTLV-II infection, while controlling for the effects of age, race, gender, drug and alcohol use, and, among the HIV-seropositive subjects, CD4 count. The RR of neurologic disability associated with HTLV-II infection compared with the uninfected group was $12.9(95 \% \mathrm{CI}$ $[3.62,45.61], p=0.0001$ ) (figure 1). Similarly, the RR of stocking/glove neuropathy associated with HTLV-II infection was 3.5 , although this did not quite attain statistical significance $(95 \%$ CI $[0.90,13.56], p=0.0696$ ) (figure 2). There were no statistically significant increases in RR associated with HIV infection alone or with co-infection for these outcomes, nor were there any statistically significant increases in RR among any of the infected subgroups for the development of extrapyramidal signs, bladder dysfunction, or cognitive impairment. Finally, the progression of co-infected subjects was not statistically significantly different from subjects infected with HTLV-II alone. Similar results were found when these analyses were repeated with the subsample frequency-matched for age.

As far as individuals initially noted to be neurologically affected are concerned, all of the uninfected subjects were rated less disabled at their last visit than at their first visit. One-third of the HIV-positive subjects were rated less disabled at their last visit than at their first visit, whereas two-thirds remained stable or progressed. Only one-fifth of the co-infected and HTLV-II-infected subjects were rated less disabled at their last visit than at their first visit, and $80 \%$ were stable or progressed. The infected subgroups (especially the HTLV-II-positive and co-infected groups) were more likely to remain stable or progress during follow-up. 


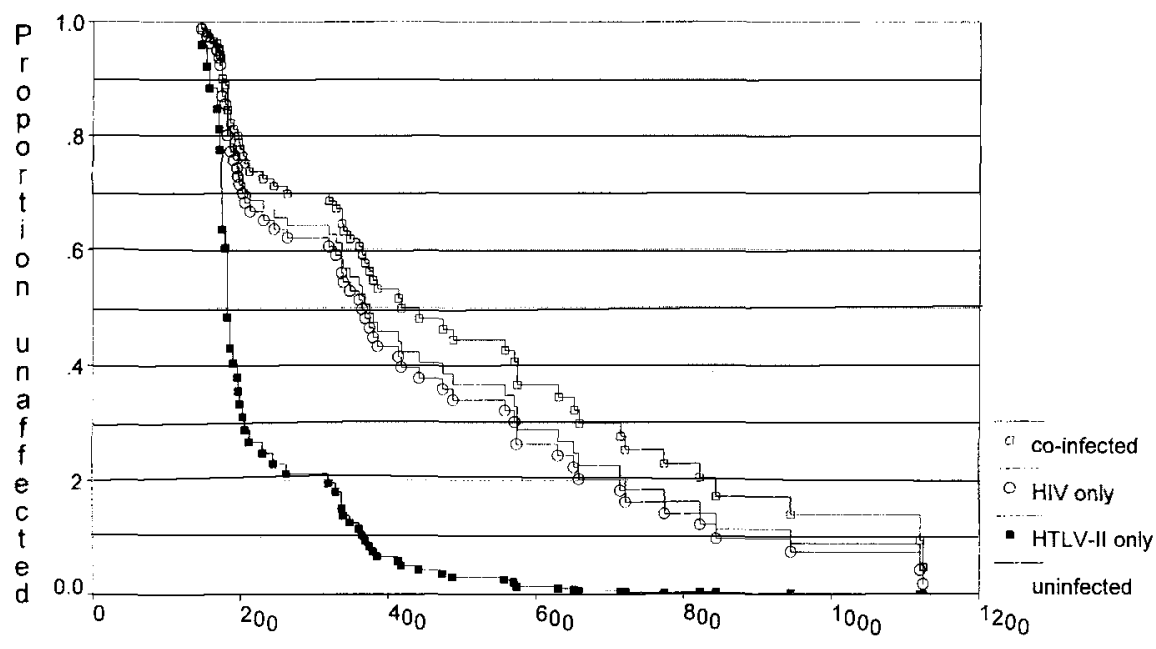

days from baseline to stocking/glove neuropathy endpoint
Figure 2. Cox proportional hazards plot showing development of stocking /glove neuropathy among members of a cohort of injection drug us. ers during follow-up.
Discussion. HTLV-II infection was present among $25 \%$ of a group of volunteer IDUs recruited for this study. HTLV-II was more frequent among AfricanAmericans and was associated with increased age and overall neurologic disability. As expected, HIV infection was also associated with increased neurologic disability and cognitive impairment. There was no evidence for an interaction between HTLV-II and HIV with respect to the frequency of these findings. Over time, only subjects infected with HTLV-II alone were more likely than uninfected subjects to develop neurologic disability, after controlling for the effects of age, race, gender, drug use, and alcohol use. The results of the current study suggest a direct HTLV-II effect on the nervous system.

The cross-sectional or prevalence results may be expected to differ from the longitudinal or incidence results if there is an association between the presence of certain findings and survival. For example, incident neurologic findings may be easier to detect in subjects more likely to survive, and this may explain the fact that $\mathrm{H}^{\prime} \mathrm{TLV}$-II was associated with incident neurologic findings while an association did not occur in HIV-infected or co-infected subjects who were more likely to die during follow-up.

While previous studies have failed to demonstrate a specific disease or symptom complex consistently associated with HTLV-II infection, the current findings from a prospectively studied cohort of IDUs add weight to earlier case reports suggesting an association between HTLV-II and neurologic disease. ${ }^{2,10}$ The current study may, in fact, underestimate the likelihood of an association between HTLV-II and neurologic disease for several reasons. First, chronic neurologic disorders were among the exclusion criteria for the natural history study. Second, the latency for the development of neurologic dysfunction after HTLV-II infection may be extremely long. This is often the case for the development of myelopathy after infection with HTLV-I, ${ }^{11}$ a virus with which HTLV-II shares approximately $60 \%$ genetic homolo- gy ${ }^{8}$ Because the duration of infection in the HTLVII-positive subjects is not known, the infection may have been relatively recent in some and might not yet have had sufficient opportunity to produce neurologic dysfunction. Third, many of the HTLV-IIinfected subjects were also infected with HIV, and there may have been competing risks: HTLV-IIrelated neurologic impairment may have been masked by the disability, loss to follow-up, or shortened survival produced by HIV-related systemic illness.

The findings noted here do not describe a distinctive syndrome attributable to HTLV-II infection. HIV causes a number of neurologic syndromes (including dementia, myelopathy, and neuropathy) and may be responsible for the bulk of the neurologic impairment noted among co-infected subjects. We only crudely controlled for the possible confounding effects of alcohol and drug use with the dichotomous "long" and "short" use variables. Finally, the small number of subjects infected solely with HTLV-II ( $\mathrm{n}=$ 13) may be insufficient to capture the neurologic pathology, if any, unique to HTLV-II infection. However, the current findings suggest an independent effect of HTLV-II in the development of neurologic impairment. Further study of neurologic impairment among IDUs with and without HTLV-II exposure might help resolve this issue.

\section{References}

1. Hjelle B. Human T-cell leukemia/ymphoma viruses: life cycle, pathogenicity, epidemiology and diagnosis. Arch Pathol Lab Med 1991;115:440-450.

2. Hjelle B, Appenzeller $O$, Mills $R$, et al. Chronic neurodegenerative disease associated with HTLV-II infection. Lancet 1992;339:645-646.

3. Lee H, Swanson P, Rosenblatt J, et al. Relative prevalence and risk factors of HTLV-I and HTLV-II infection in US blood donors. Lancet 1991;337:1435-1439.

4. Centers for Disease Control. CDC classification system for human T-lymphotropic virus type III/lymphadenopathy-associated virus infections. MMWR 1986;35:334-339. 
5. Marder K, Stern Y, Malouf R, et al. Neurologic and neuropsychological manifestations of human immunodeficiency virus infection in intravenous drug users without acquired immunodeficiency syndrome: relationship to head injury. Arch Neurol 1992;49:1169-1175.

6. Kurtzke J. Rating neurologic impairment in multiple sclerosis: an expanded disability status scale (EDSS). Neurology 1983;33:1144-1152.

7. Marder K, Liu X, Stern Y, et al. Neurological signs and symptoms in a cohort of gay men followed for 4.5 years. Neurology 1995;45:261-266

8. Chen Y-M, Lee T-H, Wiktor S, et al. Type-specific antigens for serological discrimination of H'TLV-I and H'TLV-II infection. Lancet 1990;336:1153-1155.

9. Okayama A, Korber B, Chen Y-M, et al. Unusual pattern of antibodies to human T-cell leukemia virus type-1 in family members of adult T-cell leukemia patients. Blood 1991;78:3323-3329.

10. Jacobson S, Lehkey T, Nishimura M, Robinson S, McFarlin D, Dhib-Jalbut S. Isolation of HTLV-II from a patient with chronic, progressive neurological disease clinically indistinguishable from HTLV-I-associated myelopathy/tropical spastic paraparesis. Ann Neurol 1993;33:392-396.

11. Hollsberg P, Hafler D. Pathogenesis of diseases induced by human lymphotropic virus type I infection. N Engl J Med 1993;328:1173-1182.

\title{
Aortic atheromas and acute ischemic stroke:
}

\section{A transesophageal echocardiographic study in an ethnically mixed population}

\author{
M.R. Di Tullio, MD; R.L. Sacco, MD; D. Gersony, MD; H. Nayak, MD; R.G. Weslow, MD; \\ D.E. Kargman, MD; and S. Homma, MD
}

\begin{abstract}
Article abstract-Purpose: Proximal aortic atheromas have been suggested as a potential ischemic stroke determinant in the elderly, especially in cases of unexplained (cryptogenic) stroke. Our aim was to assess the potential role of proximal aortic atheromas as an independent risk factor for stroke by comparing their frequency in patients with acute ischemic stroke and in stroke-free control subjects. The frequency of atheromas was also compared among different ethnic groups. Patients and Methods: A case-control study was conducted in 106 patients with acute ischemic stroke and 114 stroke-free control subjects. The presence of atheromas of the proximal portion of the aorta was assessed by biplane transesophageal echocardiography. Atheromas were categorized on the basis of their thickness $(0.2$ to $0.4 \mathrm{~cm}, \mathrm{small} ; \geq 0.5 \mathrm{~cm}$, large $)$ and complexity (i.e., ulceration or mobility). The association between aortic atheromas and ischemic stroke was tested, controlling for patients' demographic variables and stroke risk factors. In stroke patients, subgroup analyses were performed to test the associations between aortic atheromas and stroke diagnostic subtypes (determined cause versus cryptogenic) and presence and degree of carotid stenoses by duplex Doppler examination. Results: The frequency of large aortic atheromas was greater in stroke patients than in controls (26\% versus $13 \%$; crude odds ratio [OR] $2.4,95 \%$ CI 1.2 to 4.7); ulcerated or mobile atheromas also tended to be more frequent in stroke patients (12\% versus 5\%; OR 2.5, 95\% CI 1.0 to 6.8). Differences were entirely attributable to the subgroup of patients aged 60 years or older, in whom the frequency of ulcerated or mobile atheromas was particularly high among cryptogenic stroke patients $22 \%$ versus $8 \%$ in control subjects; OR 3.4, 95\% CI 1.1 to 11.2). Multivariate analysis showed the presence of large atheromas to be independently associated with stroke in the entire study group (adjusted OR 2.6, 95\% CI 1.1 to 5.9) and in the older subgroup (OR 2.4, $95 \% \mathrm{CI} 1.1$ to 5.7 ). Carotid stenosis $\geq 60 \%$ was more frequent with increasing size and complexity of aortic atheromas but had low predictive value (16\%) for presence of large atheromas; moreover, $36 \%$ of patients with mild or no carotid stenosis had large or complex aortic atheromas. No significant differences were found in the frequency of atheromas by ethnic group. Conclusions: Proximal aortic atheromas $\geq 0.5 \mathrm{~cm}$ in size are a risk factor for ischemic stroke in patients aged 60 years or older. Ulcerated or mobile atheromas may play a role in explaining some cryptogenic strokes in the elderly. The risk for stroke of patients with aortic atheromas may be similar across different ethnic groups. The absence of carotid stenosis does not exclude aortic atheromas as a potential cause for ischemic stroke.
\end{abstract}

NEUROLOGY 1996;46:1560-1566

The presence of protruding or ulcerated atheromas in the ascending aorta and the aortic arch is associated with an increased frequency of peripheral or cerebral embolic events. ${ }^{1-3}$ There have been suggestions that protruding aortic arch atheromas were a risk factor for ischemic stroke due to their potential

From the Departments of Medicine (Drs. Di Tullio, Nayak, Weslow, and Homma), Neurology (Drs. Sacco, Gersony, and Kargman), and Public Health (Epidemiology) (Dr. Sacco), Sergievsky Center, Columbia-Presbyterian Medical Center. New York, NY.

Supported in part by grants from the National Institute of Neurological Disorders and Stroke (R01 NS 29993 and NS 33248 , T32 NS 07153).

Presented in part at the 66th Scientific Sessions of the American Heart Association. Atlanta, Georgia, November 1993.

Received July 6, 1995. Accepted in final form September 29, 1995.

Address correspondence and reprint requests to Dr. Marco R. Di Tullio, Division of Cardiology-PH 3-342, Columbia-Presbyterian Medical Center, 630 West 168th Street, New York. NY 10032. 